\title{
ERRATUM
}

Victor V. Skakun · Mark A. Hink

Anatoli V. Digris · Ruchira Engel · Eugene G. Novikov

Vladimir V. Apanasovich · Antonie J. W. G. Visser

\section{Global analysis of fluorescence fluctuation data}

\section{Eur Biophys J (2005) 34:323-334}

We noticed a mistake in equation 4 on page 324 . The equation is printed as follows:

$$
\begin{aligned}
& G(\tau)= 1+\frac{1}{\langle N\rangle} \cdot \frac{1-T+T \mathrm{e}^{-\tau / \tau_{\text {trip }}}}{(1-T)} \sum_{j} \Phi_{j} \\
& \times \frac{1}{\left(1+\frac{\tau}{\tau}\right) \sqrt{1+\left(\frac{\omega_{x y}}{\omega_{z}}\right)^{2} \frac{\tau}{\tau} \operatorname{dif}_{, j}}} . \\
& \text { with } \Phi_{j}=\frac{\left(\eta_{j} Y_{j}^{2}\right)}{\left(\sum_{j} \eta_{j} Y_{j}\right)^{2}} .
\end{aligned}
$$

but it should be:

$$
\begin{gathered}
G(\tau)=1+\frac{1}{\langle N\rangle} \cdot \frac{1-T+T \mathrm{e}^{-\tau / \tau_{\text {trip }}}}{(1-T)} \sum_{j} \Phi_{j} \\
\times \frac{1}{\left(1+\frac{\tau}{\tau_{\mathrm{dif}, j}}\right) \sqrt{1+\left(\frac{\omega_{x y}}{\omega_{z}}\right)^{2} \frac{\tau}{\tau_{\mathrm{dif}, j}}}} \\
\text { with } \Phi_{j}=\frac{\left(\eta_{j}^{2} Y_{j}\right)}{\left(\sum_{j} \eta_{j} Y_{j}\right)^{2}}
\end{gathered}
$$

(4) stating that each species contributes to the autocorrelation curve according to the square of the brightness $\eta$ and not to the square of the molecular fraction Y.

The online version of the original article can be found at http://dx.doi.org/10.1007/s00249-004-0453-9

V. V. Skakun · A. V. Digris · V. V. Apanasovich

Department of Systems Analysis, Belarusian State University, Minsk, 220050, Belarus

M. A. Hink · R. Engel · A. J. W. G. Visser $(\bowtie)$

MicroSpectroscopy Centre, Laboratory of Biochemistry,

Wageningen University, P.O. Box 8128,

6700 ET Wageningen, The Netherlands

E-mail: ton.visser@wur.nl

Fax: + 31-317-484801

E. G. Novikov

Institut Curie, Section de Recherche, Service Bioinformatique, 26 Rue d'Ulm, 75005 Paris, France 